\title{
RWRMTN: a tool for predicting disease- associated microRNAs based on a microRNA-target gene network
}

\author{
Duc-Hau Le ${ }^{*}$ (D) and Trang T. H. Tran
}

\author{
* Correspondence: hauldhut@gmail. \\ com \\ Department of Computational \\ Biomedicine, Vingroup Big Data \\ Institute, No 7, Bang Lang 1 Street, \\ Viet Hung Ward, Long Bien District, \\ Hanoi, Vietnam
}

\begin{abstract}
Background: The misregulation of microRNA (miRNA) has been shown to cause diseases. Recently, we have proposed a computational method based on a random walk framework on a miRNA-target gene network to predict disease-associated miRNAs. The prediction performance of our method is better than that of some existing state-of-the-art network- and machine learning-based methods since it exploits the mutual regulation between miRNAs and their target genes in the miRNA-target gene interaction networks.

Results: To facilitate the use of this method, we have developed a Cytoscape app, named RWRMTN, to predict disease-associated miRNAs. RWRMTN can work on any miRNA-target gene network. Highly ranked miRNAs are supported with evidence from the literature. They then can also be visualized based on the rankings and in relationships with the query disease and their target genes. In addition, automation functions are also integrated, which allow RWRMTN to be used in workflows from external environments. We demonstrate the ability of RWRMTN in predicting breast and lung cancer-associated miRNAs via workflows in Cytoscape and other environments.

Conclusions: Considering a few computational methods have been developed as software tools for convenient uses, RWRMTN is among the first GUI-based tools for the prediction of disease-associated miRNAs which can be used in workflows in different environments.
\end{abstract}

Keywords: Disease-associated miRNAs, miRNA-target interaction, Random walk with restart, Automation, Cytoscape app, CyREST command APIs, CyREST APIs

\section{Background}

Prediction of novel disease-associated miRNAs is an important task in biomedicine. A number of computational methods, including network-based and machine learningbased ones, have been introduced for predicting disease-associated miRNAs [1-3]. However, few of them have been developed as prediction tools to facilitate their use [4, 5]. In general, disease similarity, miRNA similarity and known disease-miRNA

(c) The Author(s). 2020 Open Access This article is licensed under a Creative Commons Attribution 4.0 International License, which permits use, sharing, adaptation, distribution and reproduction in any medium or format, as long as you give appropriate credit to the original author(s) and the source, provide a link to the Creative Commons licence, and indicate if changes were made. The images or other third party material in this article are included in the article's Creative Commons licence, unless indicated otherwise in a credit line to the material. If material is not included in the article's Creative Commons licence and your intended use is not permitted by statutory regulation or exceeds the permitted use, you will need to obtain permission directly from the copyright holder. To view a copy of this licence, visit http://creativecommons.org/licenses/by/4.0/. The Creative Commons Public Domain Dedication waiver (http://creativecommons.org/publicdomain/zero/1.0/) applies to the data made available in this article, unless otherwise stated in a credit line to the data. 
associations are usually used as input data for both approaches [6]. The similarity information was represented as networks (e.g., disease similarity network, miRNA similarity network) in network-based methods, then label propagation algorithms were often used to transfer disease-miRNA labels between miRNAs and diseases via the networks [7-13]. Meanwhile, that information was often represented by matrices in some machine learning-based methods, then matrix factorization methods were used [14-17]. Network-based methods are mainly relied on homogeneous miRNA networks [10-13]. In such networks, nodes represent miRNAs and edges represent the degree of functional similarity between miRNAs. Based on these homogeneous miRNA networks, associations between miRNAs and diseases are predicted based on the assumption that functionally related miRNAs associate with phenotypically similar diseases.

A common limitation of the homogeneous miRNA network-based methods is that the biological interactions between miRNAs and their target genes might be used ineffectively. This is because those interactions were only embedded as a degree of similarity among miRNAs. For instances, the similarity between two miRNAs was measured by a number of their shared target genes [11, 18-21]. These miRNA-target interactions, including both predicted and experimentally validated ones, are now largely available in a number of miRNA-target databases [22] and become useful resources for analysis. This has inspired us to propose a novel network-based method for predicting diseaseassociated miRNA [19] using miRNA-target gene interactions. More specifically, we exploited the mutual regulation between miRNAs and their target genes to construct mutual heterogeneous miRNA-target gene interaction networks (shortly called miRNAtarget interaction networks) (i.e., nodes represent miRNAs and their target genes, and edges refer to the physical interactions between miRNAs and their target genes). We then proposed a novel method, namely RWRMTN, based on random walk with restart (RWR) algorithm on the miRNA-target interaction network to rank candidate miRNAs. Experiment results show that RWRMTN outperforms existing state-of-the-art methods including a network-based method RWRMDA [10], which also uses the RWR algorithm but on homogeneous miRNA networks, and a machine learning-based method RLSMDA [17]. RWRMTN was also proven to be stable, and it achieved relatively high performance for both experimentally validated and predicted miRNA-target gene networks [19].

Although many computational methods have been proposed for predicting diseaseassociated miRNAs, however, only a few of them have been developed as software tools which are convenient for biomedical applications. Indeed, we investigated 59 software tools for predicting disease-miRNA associations in OMICtools [5], but most of them provided only source code and with command-line interfaces, which are not convenient for most of the biologists. Ten of them provide web-based interfaces, but only a few are accessible such as CHNmiRD [23], OncomiR [24] and miRConnect [25] (Table 3 shows a comparison between RWRMTN with the ten web-based tools).

To facilitate the use of RWRMTN, we develop a tool running on the Cytoscape framework [33] to predict disease-associated miRNAs. RWRMTN can rank all miRNA candidates in the miRNA-target gene network as well as candidates provided by users. In addition, it can provide evidence from literature about associations with the disease of interest for highly ranked miRNAs. These miRNAs then can also be visualized in relationships with their target genes as well as supporting evidence. Equipped by newly- 
introduced automation features of Cytoscape, the use of RWRMTN can be extended to workflows in other environments. Functions of RWRMTN were demonstrated in predicting breast and lung cancer-associated miRNAs via workflows in Cytoscape GUI platform and other environments.

\section{Implementation}

\section{Main functions and workflow}

RWRMTN is written as a Cytoscape app (Fig. 1(a)). The main task of RWRMTN is to predict novel disease-associated miRNAs. The primary result is ranking of candidate miRNAs based on our previously proposed method RWRMTN [19] (see brief description in the next section). To make the result more reliable and intuitive, RWRMTN provides two more functions which are evidence collection and visualization. The evidence collection function gathers evidence from literature about the association of highly ranked miRNAs and the disease of interest. The visualization function builds a network of the selected miRNAs (e.g., highly ranked miRNAs) in relationships with their target genes and the disease of interest. To make RWRMTN reaches a wider range of users, we additionally implement automation features so that those functions of RWRMTN can be called using CyREST APIs and CyREST Command APIs from workflows written in other environments such as $\mathrm{R}$ and Python.

Prediction of novel disease-associated miRNAs is designed to complete through a four-step workflow (Fig. 1(b)). First, two datasets, including a miRNA-target interaction network and known disease-miRNA associations, must be specified. To facilitate the use of RWRMTN, we preinstalled some widely used datasets. However, to be flexible, the user can freely import others. Second, a disease of interest and candidate miRNAs are selected to rank. The candidates can be either all remaining miRNAs in the miRNA-target interaction network (i.e., excluding known miRNAs associated with the disease of interest) or freely inputted by the user. Third, highly ranked candidate miRNAs can be selected for an evidence search. More specifically, we searched for the cooccurrence of a candidate miRNA and the disease of interest from literature in PubMed

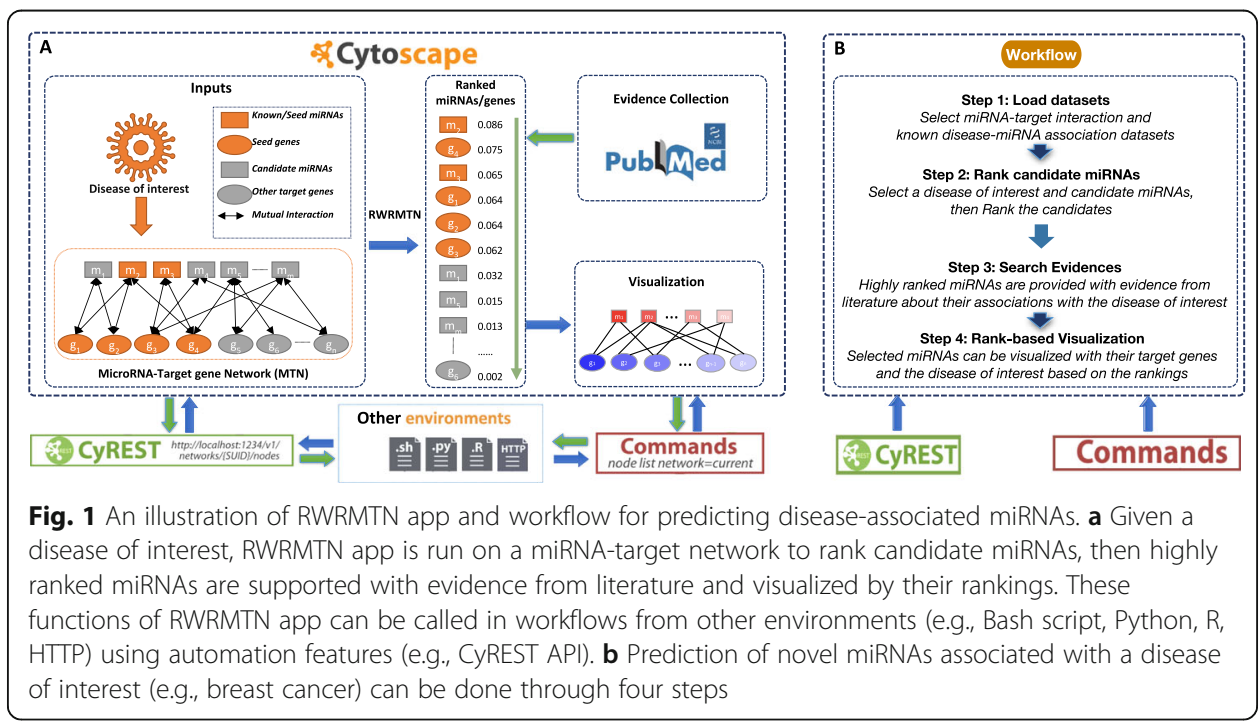


via NCBI API [34]. Thus, relevant evidence from newly published research will be retrieved each time of the evidence search. Evidenced miRNAs are provided with PubMed ID of the studies supporting an association between the miRNAs and the disease of interest. Finally, the selected miRNAs can be visualized based on their rankings and in relation with their target genes, the disease of interest and the supporting PubMed IDs.

\section{RWRMTN method}

RWR is a variant of random walk algorithm, and it mimics a walker that moves from a current node in a network (i.e., $G(V, E)$ with a set of nodes $V=\left\{v_{1}, v_{2}, \ldots, v_{N}\right\}$ and a set of links $E=\left\{\left(v_{\dot{v}} v_{j}\right) \mid v_{\dot{v}}, v_{j} \in V\right\}$, a set of seed nodes $\left.S \subseteq V\right)$ to a randomly selected adjacent node or goes back to source nodes (also called seed nodes) with a restart-probability $(\gamma)$.

$$
p_{t+1}=(1-\gamma) W^{\prime} p_{t}+\gamma p_{0}
$$

where:

- $W^{\prime}$ represents a transition probability matrix of the network

- $p_{\mathrm{t}}$ is a $|V| \times 1$ probability vector of $|V|$ nodes at a time step $t$ of which the $i$ th element represents the probability of the walker being at node $v_{i} \in V$.

$-p_{0}$ is the $|V| \times 1$ initial probability vector.

In RWRMDA method [10], the RWR algorithm was used to rank miRNAs in homogeneous miRNA networks; therefore, the set of seed nodes $(S)$ only contains known disease miRNA $\left(S_{m}\right)$ (i.e., $\left.S=S_{m}\right)$.

$$
\left(p_{0}\right)_{i}=\left\{\begin{array}{c}
\frac{1}{\left|S_{m}\right|} \text { ifv } v_{i} \in S_{m} \\
0 \text { otherwise }
\end{array}\right.
$$

In RWRMTN [19], we assumed that the mutual regulation between a miRNA and their targets leads to a transfer of disease information between them. Therefore, we force the RWR algorithm to start from a set of seed nodes which consists of not only known disease miRNAs but also their target genes. In particular, we enlarge the set of seed node $(S)$ by adding target genes $\left(S_{g}\right)$ of the known disease miRNAs (i.e., $S=S_{m} \cup S_{g}$ ), with $\alpha \in(0,1)$ is a weight parameter, which controls the disease information transferred between miRNAs and their target genes.

$$
\left(p_{0}\right)_{i}=\left\{\begin{array}{c}
\alpha \frac{1}{\left|S_{m}\right|} \text { if } v_{i} \in S_{m} \\
(1-\alpha) \frac{1}{\left|S_{g}\right|} \text { if } v_{i} \in S_{g} \\
0 \text { otherwise }
\end{array}\right.
$$

For both methods, all miRNAs in the network are eventually ranked according to the steady-state probability vector $p_{\infty}$, which is obtained by repeating the iterations until convergence is reached (in this study, $\left\|p_{\mathrm{t}+1}-p_{\mathrm{t}}\right\|<10^{-6}$ ).

In the previous study, we have investigated the effects of the restart-probability $(\gamma)$ and the weight parameter $(\alpha)$ on the prediction performance of RWRMTN on two 
miRNA-target gene networks. More specifically, we varied the weight parameter $(\alpha)$ in the range $\{0.1,0.3,0.5,0.7,0.9\}$ and the restart probability $\gamma$ in the range $[0.1,0.9]$ in steps of 0.1. Experimental results showed that the performance of RWRMTN slightly increased according to the change of the weight parameter in both the networks. This indicates that disease information contained in known disease miRNAs is still more important than that in their target genes when ranking candidate disease-associated miRNAs. In addition, RWRMTN is either slightly better or stable when the restart probability $\gamma$ increased in the two networks. The slight increase in the prediction performance in a network suggested that disease miRNAs in that network are less modularized than the other.

\section{Preinstalled data}

To facilitate the use of the app, we preinstalled some datasets. First, two miRNA-target gene networks collected from an experimentally validated dataset, miRWalk [35] and a predicted dataset, TargetScan [36] were preinstalled (Table 1). However, the user can import any other miRNA-target gene network to use. Second, two known diseasemiRNA association datasets were also pre-collected, i.e., miR2Disease [37] and HMDD [38] (Table 2).

\section{Results}

The overall prediction performance of RWRMTN on a set of diseases was reported in our previous study [19]. In this section, the effectiveness of RWRMTN was demonstrated for breast cancer in two case studies via different scenarios. Given the breast cancer with their known associated miRNAs, firstly, all other miRNAs in the miRNAtarget networks were used as candidates. The overall performance of RWRMTN in terms of AUC (Area under the ROC curve) for breast cancer was assessed using a leave-one-out cross-validation (LOOCV) scheme (Fig. 2). Then, the candidates were ranked and supported with evidence. More specifically, we performed this task with candidate miRNAs from the miRNA-target gene network constructed from TargetScan [36] based on 31 known breast cancer-associated miRNAs reported in miR2Disease [37] using Cytoscape menu and Command APIs via the four-step workflow (Fig. 3). As a result, four of top 10 miRNA candidates were supported with evidence from literature about their associations with breast cancer. Secondly, only miRNAs that were differentially expressed between case and control samples were used as the candidates for ranking. More specifically, we ranked 799 miRNAs that were differentially expressed between the 64 wild-type samples and 36 TP53 mutant breast cancer samples collected from [39] via CyREST API called in R environment using the miRNA-target gene network constructed from miRWalk [35] and the known disease-miRNA association dataset HMDD [38]. The scripts for calling the CyREST API in other environments such as

Table 1 Preinstalled miRNA-target gene interaction datasets

\begin{tabular}{llll}
\hline Datasets & Number of miRNAs & Number of target genes & Number of known interactions \\
\hline miRWalk & 740 & 11,976 & 38,569 \\
TargetScan & 1537 & 15,031 & 520,256 \\
\hline
\end{tabular}


Table 2 Preinstalled known disease-miRNA association datasets

\begin{tabular}{llll}
\hline Datasets & Number of miRNAs & Number of diseases & Number of known associations \\
\hline miR2Disease & 118 & 53 & 270 \\
HMDD & 574 & 243 & 5618 \\
\hline
\end{tabular}

Python and Bash were also introduced. Furthermore, another case study on lung cancer was demonstrated (See more detail in Additional file 1).

\section{Comparison between RWRMTN with other tools}

We investigated 59 software tools (as of 16th March 2020) for predicting diseasemiRNA associations in OMICtools [5] which has accumulated tens of thousands of tools for omics data. However, most of them provide only source code and with command-line interfaces, which are not convenient for most of the biologists. Indeed, 14 out of the 59 tools are web-based including: mirfluence [26], Ifmda [27], CHNmiRD [23], miRiaD [28], OncomiR [24], miRPD [40], MIDP [30], DISMIRA [31], MDHGI [32], CMP [29], miRConnect [25], DMPred [41], MMiRNA-Tar [42] and miRNACon [43]. However, the web page of DMPred [41] could not be found, and miRPD [40] was actually designed for identifying microRNAs from deep sequencing data. In addition, MMiRNA-Tar [42] and miRNACon [43] were not designed specifically for predicting disease-associated miRNAs. Thus, the four tools were eliminated from the comparison. Thus, we finally have compared the rest (ten tools) with RWRMTN (Table 3).

In the aspect of function comparison to RWRMTN, as tools for predicting diseaseassociated miRNAs, all of them provided ranking for candidate miRNAs, however only four of them provided evidence for potential disease-miRNA associations. RWRMTN, on the other hand, integrated NCBI API [34] to the evidence search function, thus relevant evidence from recently published research will be retrieved each time users perform the task. Besides displaying the results in tabular form, only two tools visualized
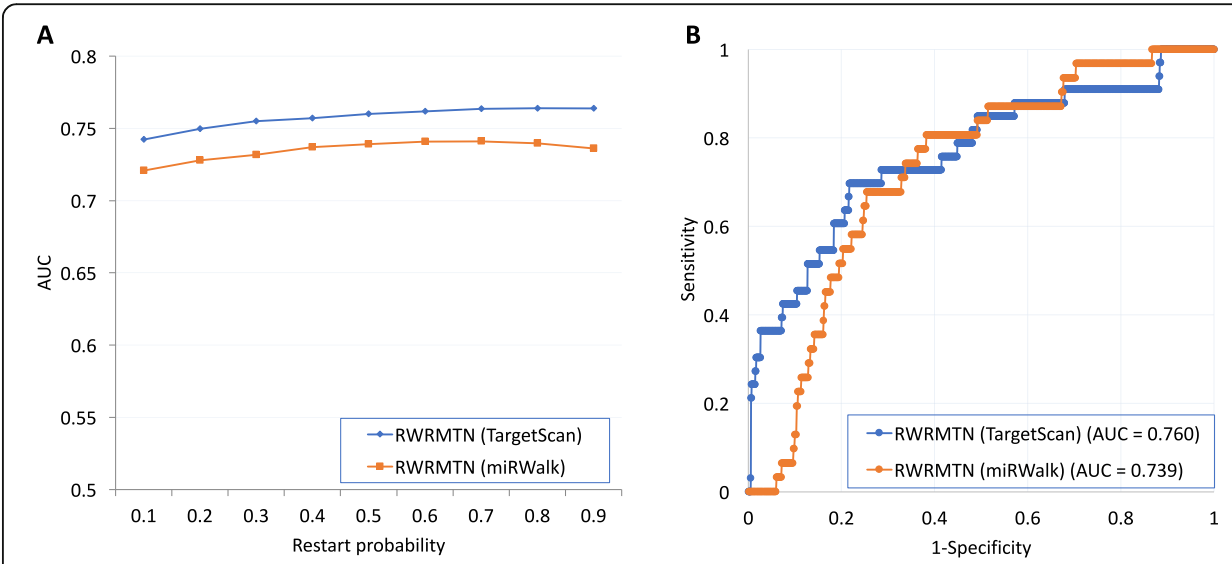

Fig. 2 Prediction performance of breast cancer by RWRMTN on the two miRNA-target gene datasets (miRWalk and TargetScan). a The AUC values when the restart probability was varied in a range $[0.1,0.9]$. b The ROC curve and the AUC values when the restart probability was set to 0.5 for the case study. For all experiments, the weight parameter was set to 0.5 


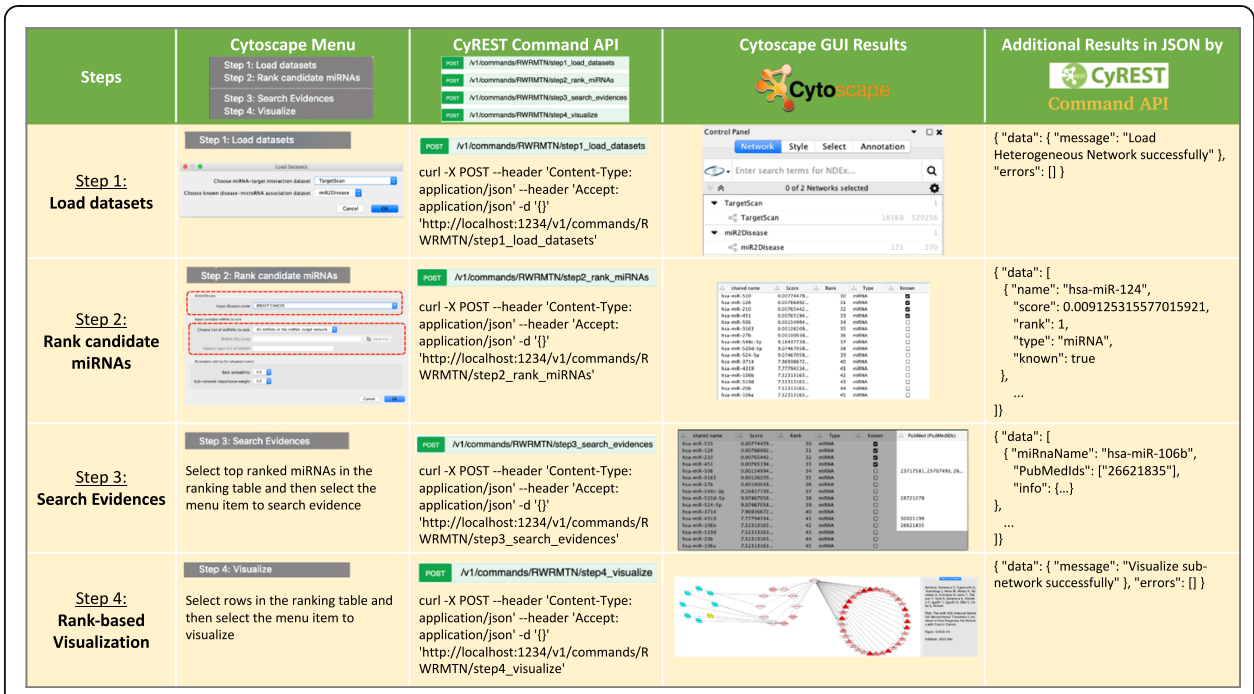

Fig. 3 A four-step workflow of RWRMTN for predicting breast cancer-associated miRNAs. Step 1: Load datasets: a miRNA-target interaction dataset and a known disease-miRNA association dataset must be selected. Step 2: Rank candidate miRNAs: a disease of interest (e.g., breast cancer) is chosen, then a set of candidate miRNAs is specified and ranked. Step 3: Search Evidences: Top-ranked candidate miRNAs are selected and provided with evidence from literature about their associations with the disease of interest. Step 4: Rank-based visualization: the selected candidate miRNAs are visualized based on their rankings and their relationships with the disease of interest, known breast cancer-associated miRNAs and supporting PubMed IDs. These steps can be performed by either the Cytoscape menu or CyREST Command APIs called from other environments (e.g., R)

the association between miRNAs and diseases. In contrast, highly ranked miRNAs, known miRNAs, target genes, the disease of interest as well as supporting PubMed IDs and their associations could be intuitively viewed in a network in RWRMTN. Lastly, none of them provides automation function of reproducibility to different environments. Equipped with CyREST API of Cytoscape platform, RWRMTN functions can be invoked from any workflows running in other environments. In other aspects, like most of the tools, RWRMTN was also designed for multiple diseases. However, it is more flexible in providing the input data, since users can opt between preinstalled datasets or their own. None of the web-based tools provided this function. Finally, only three out of the ten web-based tools can be accessed via links provided in their publications. In contrast, RWRMTN is easy to be managed and maintained since the available update of RWRMTN will be notified by Cytoscape.

\section{Prediction of breast cancer-associated miRNAs using Cytoscape menu and CyREST command API}

In this section, we demonstrate the use of RWRMTN in predicting novel breast cancer-associated miRNAs by the four-step workflow. The workflow can be accomplished using either Cytoscape menu or CyREST Command API (Fig. 4) (See more detail in Additional file 1).

For the first step, a miRNA-target interaction dataset TargetScan [36] and a known disease-miRNA association dataset miR2Disease [37] were used. Note that, besides the 


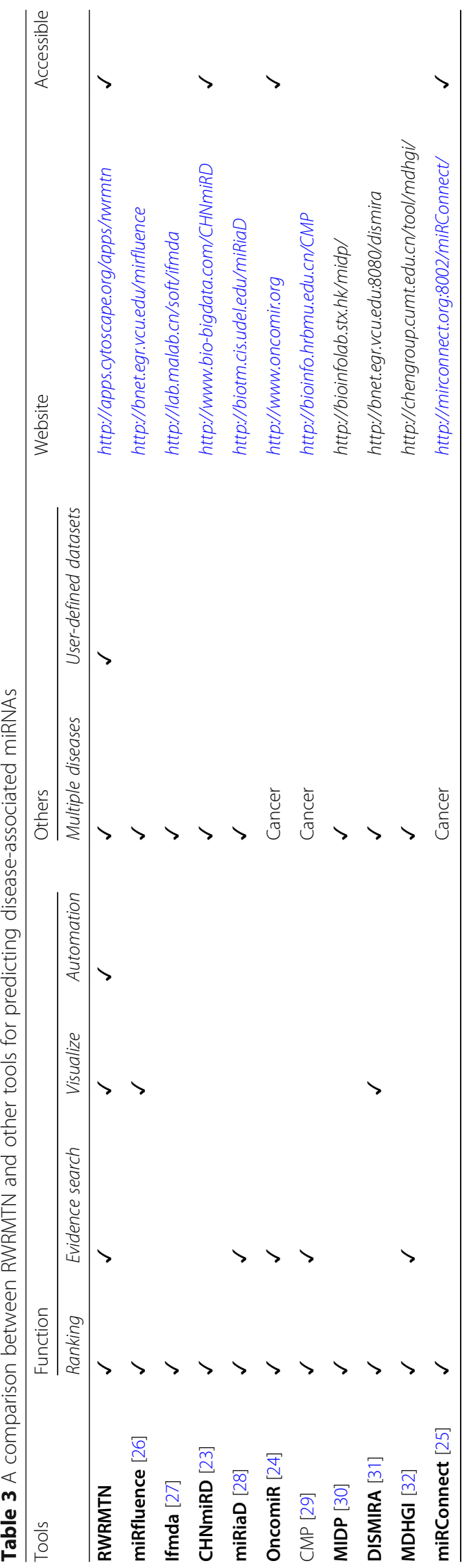



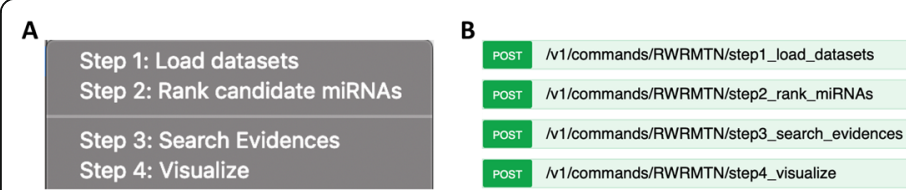

Step 1: Load Datasets

Step 2: Rank candidate miRNAs

Step 4: Visualize

/v1/commands/RWRMTN/step4_visualize

Step 2: Rank candidate miRNAs

Step 3: Search Evidences

Step 4: Visualize

Fig. 4 Perform the four-step workflow of RWRMTN. a via Cytoscape menu (Apps $\rightarrow$ RWRMTN). b via CyREST Command APIs (Help $\rightarrow$ Automation $\rightarrow$ CyREST Command API $\rightarrow$ RWRMTN)

two preinstalled datasets, miRNA-target interaction network can be freely imported by the user.

For the second step, a set of candidate miRNAs for breast cancer (OMIM ID: 114480 ) is ranked. To this end, we first selected the disease, then all miRNAs in the selected miRNA-target interaction network were selected as candidates for ranking. Note that, users can manually input candidate miRNAs or import them from a file. After that, the candidate miRNAs were ranked by the RWRMTN method [19] with default parameter settings (See more detail in Additional file 1).

For the third step, we selected the top ten ranked candidate miRNAs, then find the evidence of their associations with breast cancer from literature (PubMed). As a result, four of them were supported with evidence from literature about their associations with breast cancer (Fig. 5).

For example, hsa-miR-506 was supported by five studies (PubMed IDs: 23717581, 25707493, 26059632, 26398880 and 27542202). The study (PubMed ID: 23717581) [44] showed that has-miR-506 regulates epithelial-mesenchymal transition in breast cancer cell lines. Meanwhile, the study (PubMed ID: 26059632) [45] proved notable inhibition of hsa-miR-506 over-expression to proliferation and metastasis of breast cancer cells. In addition, study (PubMed ID: 26398880) [46] indicated that the mechanism underlying miRNA-506 is a contributing factor in breast carcinogenesis (has-miR-506 was proven to be a tumor suppressor). In addition, hsa-miR-520d-5p supported by a study (PubMed ID: 28721278) [47]. More specifically, it was reported that this miRNA upregulates the activation of BRCA1 (breast cancer 1, early onset) in the DNA repair process - 35 days after transfection. Moreover, hsa-miR-4319 was showed in study PubMed ID: 30021199 as a suppressor of the malignancy of triple-negative breast cancer by regulating self-renewal and tumorigenesis of stem cells. Finally, hsa-miR-106b was proven by the experiment carried on patient samples and cell lines in the study (PubMed ID: 26621835) [48].

\begin{tabular}{|c|c|c|c|c|c|c|c|}
\hline As shared name & name & A Score & A & Rank & $\therefore$ Type & A Known & PubMed (PudMedIDs) \\
\hline hsa-miR-510 & & $0.00774478 \ldots$ & & 30 & miRNA & $\nabla$ & \\
\hline hsa-miR-126 & & $0.00766892 \ldots$ & & 31 & miRNA & $\nabla$ & \\
\hline hsa-miR-210 & & $0.00765442 \ldots$ & & 32 & miRNA & 0 & \\
\hline hsa-miR-451 & & $0.00765194 \ldots$ & & 33 & miRNA & $\nabla$ & \\
\hline hsa-miR-506 & & $0.00154994 \ldots$ & & 34 & miRNA & 口 & $23717581,25707493,26059632,26398 \ldots$ \\
\hline hsa-miR-3163 & & $0.00126209 \ldots$ & & 35 & miRNA & 口 & \\
\hline hsa-miR-27b & & $0.00100038 \ldots$ & & 36 & miRNA & 口 & \\
\hline hsa-miR-548c-3p & & $9.16437739 \ldots$ & & 37 & miRNA & 口 & \\
\hline hsa-miR-520d-5p & & $9.07467058 \ldots$ & & 38 & miRNA & 口 & 28721278 \\
\hline hsa-miR-524-5p & & $9.07467058 \ldots$ & & 39 & miRNA & $\square$ & \\
\hline hsa-miR-3714 & & $7.96936672 \ldots$ & & 40 & miRNA & $\square$ & \\
\hline hsa-miR-4319 & & $7.77794334 \ldots$ & & 41 & miRNA & 口 & 30021199 \\
\hline hsa-miR-106b & & $7.52313163 \ldots$ & & 42 & miRNA & $\square$ & 26621835 \\
\hline hsa-miR-519d & & $7.52313163 \ldots$ & & 43 & miRNA & $\square$ & \\
\hline hsa-miR-20b & & $7.52313163 \ldots$ & & 44 & miRNA & $\square$ & \\
\hline hsa-miR-106a & & $7.52313163 \ldots$ & & 45 & miRNA & $\square$ & \\
\hline
\end{tabular}

Fig. 5 Ranked candidate miRNAs. Top-ranked candidate miRNAs were selected and provided with evidence from the literature (highlighted part) 
For the final step, the top selected candidate miRNAs can be visualized in a network based on the rankings. In addition, target genes, the disease of interest and detail information of PubMed IDs collected from Step 3 such as paper title, author list, journal name can be displayed aside in this network (Fig. 6).

\section{Prediction of breast cancer-associated miRNAs by calling CyREST API}

In this section, we first introduce some developed CyREST APIs, which provides some helpful functions. Second, we demonstrate their use in a workflow in $\mathrm{R}$ environment.

A total of four CyREST APIs were developed in RWRMTN (Fig. 7): First, GET /RWRMTN/v1/diseaseList returns a list of all diseases (OMIM ID and disease name) available in the selected known disease-miRNA association database (e.g., HMDD [38]). Based on this list, users can select a disease of interest. Second, GET /RWRMTN/v1/ diseaseList/\{diseaseName\} provides a list of diseases whose names match the query \{diseaseName\} parameter (e.g., breast). This API helps user narrow down the list of diseases to the disease of interest (e.g., OMIM ID 114480 for breast cancer). Third, POST /RWRMTN/v1/rank lets RWRMTN rank candidate miRNAs. Finally, GET:/ RWRMTN/v1/getRank/\{limit\} returns top-ranked miRNAs by setting \{limit\} parameter.

In this case study, we used a dataset GSE19783 from a study [39] published in NCBI GEO [49], which was created using Agilent-019118 Human miRNA Microarray 2.0 G4470B platform (GPL8227) and Agilent-014850 Whole Human Genome Microarray 4x44K G4112F (GPL6480) (https://www.ncbi.nlm.nih.gov/geo/query/acc.cgi?acc=GSE1 9783). The study characterizes breast cancer subtypes from joint analysis of high throughput miRNA (using GPL8227) and mRNA (using GPL6480) data.

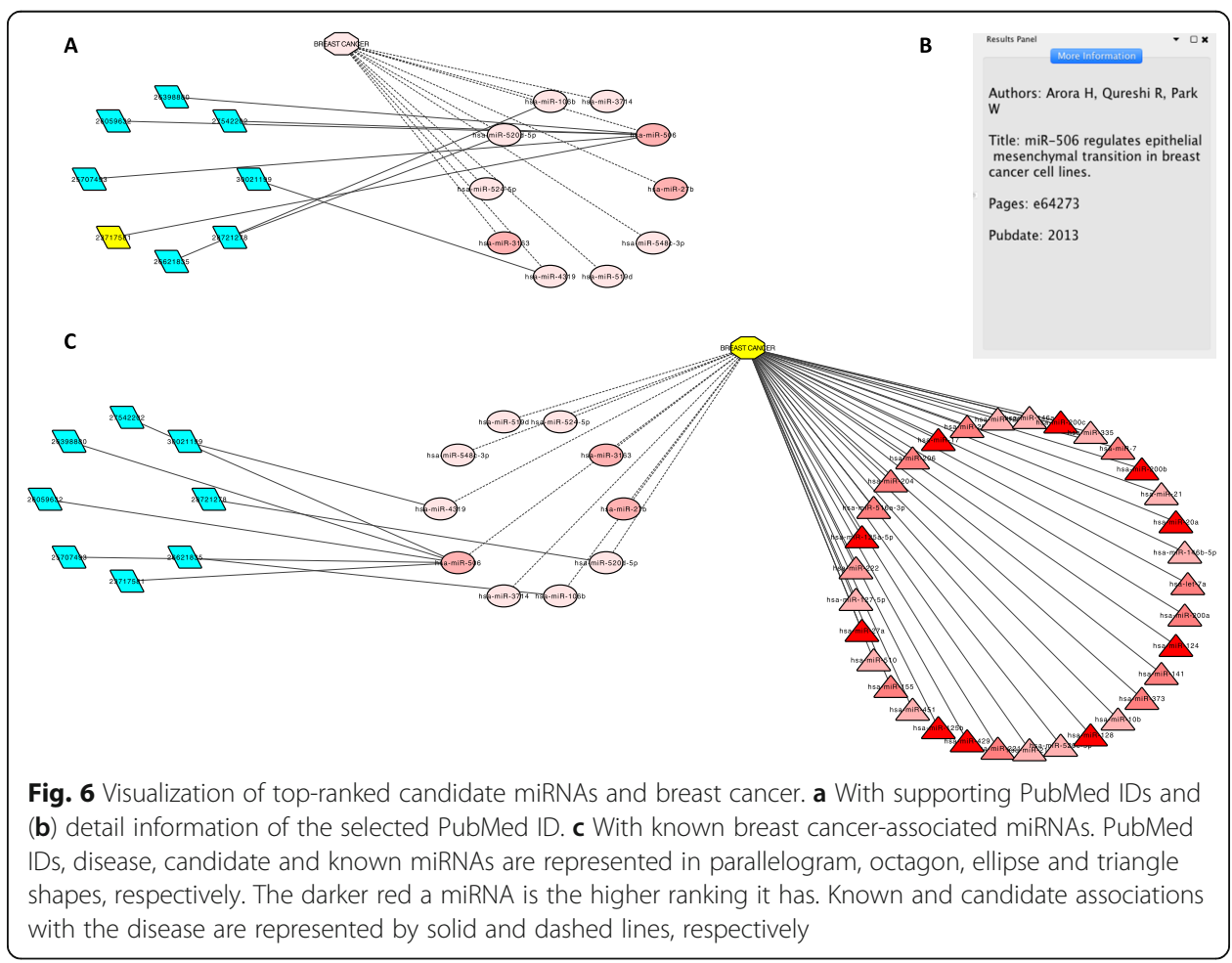




\begin{tabular}{|c|c|c|c|}
\hline \multicolumn{2}{|c|}{ Apps: RWRMTN } & Show/Hide | List Operations & Expand Operations \\
\hline GET & /RWRMTN/v1/diseaseList/\{diseaseName\} & List the diseases $r$ & natch keyword search \\
\hline GET & /RWRMTN/v1/getRank/\{limit\} & Retu & in top ranked miRNAs \\
\hline GET & /RWRMTN/v1/diseaseList & & List all diseases \\
\hline POST & /RWRMTN/v1/rank & & nk candidate miRNAs \\
\hline
\end{tabular}

In this case study, we identified 799 candidate miRNAs which were differentially expressed between the 64 wild-type samples (WT) and 36 TP53 mutant samples. Then, we ranked the candidate miRNAs by RWRMTN via a CyREST API using a miRNAtarget interaction dataset miRWalk [35] and a known disease-miRNA association dataset HMDD [38] via workflow in R environment using CyREST API POST /RWRMTN/ v1/rank (See more detail in Additional file 1).

\section{Conclusions}

In this study, we introduce a tool as a Cytoscape app, RWRMTN, for predicting novel disease-associated miRNAs. The tool was developed based on our previously proposed method with the same name, which was proven to be better than other state-of-the-art methods on overall prediction performance and to have the ability in predicting novel miRNAs associated with 23 diseases [19]. Because the core method is a network-based, thus RWRMTN can exploit network integration and visualization functions of Cytoscape. In particular, the tool relies on miRNA-target gene networks to rank candidate miRNAs with supporting functions such as evidence collection and visualization for highly ranked candidate miRNAs. In addition, by implementing automation functions, it can be used in workflows in other environments. We further demonstrate the use of RWRMTN by showing its ability in predicting novel breast and lung cancer-associated miRNAs.

\section{Availability and requirements}

Project name: RWRMTN

Project home page: https://github.com/hauldhut/RWRMTN

Operating system(s): Mac OS, Linux, Windows

Programming language: Java

Other requirements: JDK 1.8 or higher, Cytoscape 3.x or higher

License: Apache License

Any restrictions to use by non-academics: licence needed

\section{Supplementary information}

Supplementary information accompanies this paper at https://doi.org/10.1186/s12859-020-03578-3.

Additional file 1. User manual \& Case studies. 
Acknowledgements

Not applicable.

\section{Authors' contributions}

DHL conceived of the study, developed the core algorithm, TTHT developed the Cytoscape app. DHL wrote the manuscript, TTHT wrote the User manual. DHL and TTHT both wrote the case studies and approved the final manuscript.

\section{Funding}

This research is funded by Vietnam National Foundation for Science and Technology Development (NAFOSTED) under grant number 102.01-2017.14.

\section{Availability of data and materials}

RWRMTN is distributed as a Cytoscape app, and can be downloaded freely at Cytoscape App https://apps.cytoscape. org/apps/rwrmtn for non-commercial use.

\section{Ethics approval and consent to participate}

Not applicable.

\section{Consent for publication}

Not applicable.

\section{Competing interests}

The authors declare that they have no competing interests.

Received: 4 October 2019 Accepted: 1 June 2020

Published online: 15 June 2020

\section{References}

1. Zeng X, Zhang $X$, Zou Q. Integrative approaches for predicting microRNA function and prioritizing disease-related microRNA using biological interaction networks. Brief Bioinform. 2016;17(2):193-203.

2. Chen X, Xie D, Zhao Q, You Z-H. MicroRNAs and complex diseases: from experimental results to computational models. Brief Bioinform. 2017;20:bbx130.

3. Huang Z, Liu L, Gao Y, Shi J, Cui Q, Li J, Zhou Y. Benchmark of computational methods for predicting microRNA-disease associations. Genome Biol. 2019;20(1):202.

4. Wang C, Sun H, Chen L, Heikkinen L, Yang Y, Wong G. Trends in the development of miRNA bioinformatics tools; 2018.

5. Henry VJ, Bandrowski AE, Pepin A-S, Gonzalez BJ, Desfeux A. OMICtools: an informative directory for multi-omic data analysis. Database. 2014;2014. https://academic.oup.com/database/article/doi/10.1093/database/bau069/2634692.

6. Le D-H. A general computational framework for prediction of disease-associated non-coding RNAs. VNU J Sci Comput Sci Commun Eng. 2019;35(2). http://www.jcsce.vnu.edu.vn/index.php/jcsce/article/view/224.

7. Chen X, Zhang D-H, You Z-H. A heterogeneous label propagation approach to explore the potential associations between miRNA and disease. J Transl Med. 2018;16(1):348.

8. Li G, Luo J, Xiao Q, Liang C, Ding P. Predicting microRNA-disease associations using label propagation based on linear neighborhood similarity. J Biomed Inform. 2018;82:169-77.

9. Yu S-P, Liang C, Xiao Q, Li G-H, Ding P-J, Luo J-W. MCLPMDA: a novel method for miRNA-disease association prediction based on matrix completion and label propagation. J Cell Mol Med. 2019;23(2):1427-38.

10. Chen X, Liu M-X, Yan G-Y. RWRMDA: predicting novel human microRNA-disease associations. Mol BioSyst. 2012;8(10): 2792-8.

11. Le D-H. Network-based ranking methods for prediction of novel disease associated microRNAs. Comput Biol Chem. 2015:58:139-48.

12. Xuan P, Han K, Guo M, Guo Y, Li J, Ding J, Liu Y, Dai Q, Li J, Teng Z, et al. Prediction of microRNAs associated with human diseases based on weighted k most similar neighbors. PLoS One. 2013;8(8):e70204.

13. Chen $\mathrm{H}$, Zhang Z. Similarity-based methods for potential human microRNA-disease association prediction. BMC Med Genet. 2013;6(1):12.

14. Gao Z, Wang Y-T, Wu Q-W, Ni J-C, Zheng C-H. Graph regularized L2,1-nonnegative matrix factorization for miRNAdisease association prediction. BMC Bioinformatics. 2020;21(1):61.

15. Chen X, Li S-X, Yin J, Wang C-C. Potential miRNA-disease association prediction based on kernelized Bayesian matrix factorization. Genomics. 2020;112(1):809-19.

16. Gao Y-L, Cui Z, Liu J-X, Wang J, Zheng C-H. NPCMF: nearest profile-based collaborative matrix factorization method for predicting miRNA-disease associations. BMC Bioinformatics. 2019;20(1):353.

17. Chen X, Yan G-Y. Semi-supervised learning for potential human microRNA-disease associations inference. Sci Rep. 2014; 4:5501.

18. Jiang $Q$, Hao Y, Wang G, Juan L, Zhang T, Teng M, Liu Y, Wang Y. Prioritization of disease microRNAs through a human phenome-microRNAome network. BMC Syst Biol. 2010;4(Suppl 1):S2.

19. Le D-H, Verbeke L, Son LH, Chu D-T, Pham V-H. Random walks on mutual microRNA-target gene interaction network improve the prediction of disease-associated microRNAs. BMC Bioinformatics. 2017;18(1):479.

20. Le D-H. Disease phenotype similarity improves the prediction of novel disease-associated microRNAs. In: Information and Computer Science (NICS), 2015 2nd National Foundation for Science and Technology Development Conference on: 16-18 Sept. 2015; 2015. p. 76-81.

21. Le D-H, Marchal K. Integration of miRNA-miRNA networks improves the prediction of novel disease associated miRNAs, In: The First NAFOSTED Conference on Information and Computer Science; Hanoi; 2014. p. 438-48. 
22. Tan Gana NH, Victoriano AFB, Okamoto T. Evaluation of online miRNA resources for biomedical applications. Genes Cells. 2012;17(1):11-27.

23. Shi H, Zhang G, Zhou M, Cheng L, Yang H, Wang J, Sun J, Wang Z. Integration of multiple genomic and phenotype data to infer novel miRNA-disease associations. PLoS One. 2016;11(2):e0148521.

24. Wong NW, Chen Y, Chen S, Wang X. OncomiR: an online resource for exploring pan-cancer microRNA dysregulation. Bioinformatics. 2017;34(4):713-5.

25. Hua Y, Duan S, Murmann AE, Larsen N, Kjems J, Lund AH, Peter ME. miRConnect: identifying effector genes of miRNAs and miRNA families in cancer cells. PLoS One. 2011;6(10):e26521.

26. Nalluri JJ, Rana P, Barh D, Azevedo V, Dinh TN, Vladimirov V, Ghosh P. Determining causal miRNAs and their signaling cascade in diseases using an influence diffusion model. Sci Rep. 2017;7(1):8133.

27. Liu Y, Zeng X, He Z, Zou Q. Inferring MicroRNA-disease associations by random walk on a heterogeneous network with multiple data sources. IEEE/ACM Trans Comput Biol Bioinform. 2017;14(4):905-15.

28. Gupta S, Ross KE, Tudor CO, Wu CH, Schmidt CJ, Vijay-Shanker K. miRiaD: a text mining tool for detecting associations of microRNAs with diseases. J Biomed Semantics. 2016;7(1):9.

29. Li X, Wang $Q$, Zheng Y, Lv S, Ning S, Sun J, Huang T, Zheng $Q$, Ren $H$, Xu J, et al. Prioritizing human cancer microRNAs based on genes' functional consistency between microRNA and cancer. Nucleic Acids Res. 2011;39(22):e153.

30. Xuan P, Han K, Guo Y, Li J, Li X, Zhong Y, Zhang Z, Ding J. Prediction of potential disease-associated microRNAs based on random walk. Bioinformatics. 2015;31(11):1805-15.

31. Nalluri JJ, Kamapantula BK, Barh D, Jain N, Bhattacharya A, de Almeida SS, Juca Ramos RT, Silva A, Azevedo V, Ghosh P. DISMIRA: prioritization of disease candidates in miRNA-disease associations based on maximum weighted matching inference model and motif-based analysis. BMC Genomics. 2015;16(5):S12.

32. Chen X, Yin J, Qu J, Huang L. MDHGl: matrix decomposition and heterogeneous graph inference for miRNA-disease association prediction. PLOS Comput Biol. 2018;14(8):e1006418.

33. Shannon P, Markiel A, Ozier O, Baliga NS, Wang JT, Ramage D, Amin N, Schwikowski B, Ideker T. Cytoscape: a software environment for integrated models of biomolecular interaction networks. Genome Res. 2003;13(11):2498-504.

34. Sayers EW, Beck J, Brister JR, Bolton EE, Canese K, Comeau DC, Funk K, Ketter A, Kim S, Kimchi A, et al. Database resources of the National Center for Biotechnology Information. Nucleic Acids Res. 2019;48(D1):D9-D16.

35. Dweep H, Sticht C, Pandey P, Gretz N. miRWalk - database: prediction of possible miRNA binding sites by "walking" the genes of three genomes. J Biomed Inform. 2011;44(5):839-47.

36. Lewis BP, Shih I, Jones-Rhoades MW, Bartel DP, Burge CB. Prediction of mammalian MicroRNA targets. Cell. 2003;115(7): 787-98.

37. Jiang $Q$, Wang $Y$, Hao Y, Juan L, Teng M, Zhang X, Li M, Wang G, Liu Y. miR2Disease: a manually curated database for microRNA deregulation in human disease. Nucleic Acids Res. 2009;37(suppl 1):D98-D104.

38. Li Y, Qiu C, Tu J, Geng B, Yang J, Jiang T, Cui Q. HMDD v2.0: a database for experimentally supported human microRNA and disease associations. Nucleic Acids Res. 2014;42(D1):D1070-4.

39. Enerly E, Steinfeld I, Kleivi K, Leivonen S-K, Aure MR, Russnes HG, Rønneberg JA, Johnsen H, Navon R, Rødland E, et al. miRNA-mRNA integrated analysis reveals roles for miRNAs in primary breast tumors. PLoS One. 2011;6(2):e16915.

40. Sun X, Zhang J, Li A, Yuan X. mirPD: a pattern-based approach for identifying microRNAs from deep sequencing data. Digit Signal Process. 2013;23(6):1887-96.

41. Xuan $P$, Li L, Zhang T, Zhang Y, Song Y. Prediction of disease-related microRNAs through integrating attributes of microRNA nodes and multiple kinds of connecting edges. Molecules. 2019;24(17):3099.

42. Liu Y, Baker S, Jiang H, Stuart G, Bai Y. Correlating bladder cancer risk genes with their targeting MicroRNAs using MMiRNA-tar. Genomics Proteomics Bioinformatics. 2015;13(3):177-82

43. Meder B, Backes C, Haas J, Leidinger P, Stähler C, Großmann T, Vogel B, Frese K, Giannitsis E, Katus HA, et al. Influence of the confounding factors age and sex on MicroRNA profiles from peripheral blood. Clin Chem. 2014;60(9):1200-8.

44. Arora H, Qureshi R, Park W-Y. miR-506 regulates epithelial Mesenchymal transition in breast cancer cell lines. PLoS One. 2013;8(5):e64273.

45. Yu F, Lv M, Li D, Cai H, Ma L, Luo Q, Yuan X, Lv Z. MiR-506 over-expression inhibits proliferation and metastasis of breast cancer cells. Med Sci Monit. 2015;21:1687.

46. Sun G, Liu Y, Wang K, Xu Z. miR-506 regulates breast cancer cell metastasis by targeting IQGAP1. Int J Oncol. 2015;47(5): 1963-70.

47. Ishihara Y, Tsuno S, Ping B, Ashizaki T, Nakashima M, Miura K, Miura Y, Yamashita T, Hasegawa J, Miura N. Hsa-miR-520d$5 p$ promotes survival in human dermal fibroblasts exposed to a lethal dose of UV irradiation. NPJ Aging Mech Dis. 2016: 2:16029.

48. Luo Z-I, Luo H-j, Fang C, Cheng L, Huang Z, Dai R, Li K, Tian F-Z, Wang T, Tang L-J. Negative correlation of ITCH E3 ubiquitin ligase and miRNA-106b dictates metastatic progression in pancreatic cancer. Oncotarget. 2016;7(2):1477.

49. Barrett T, Wilhite SE, Ledoux P, Evangelista C, Kim IF, Tomashevsky M, Marshall KA, Phillippy KH, Sherman PM, Holko M, et al. NCBI GEO: archive for functional genomics data sets—update. Nucleic Acids Res. 2012;41(D1):D991-5.

\section{Publisher's Note}

Springer Nature remains neutral with regard to jurisdictional claims in published maps and institutional affiliations. 